Review

\title{
Level of Inclusiveness of People with Disabilities in Ghanaian Health Policies and Reports: A Scoping Review
}

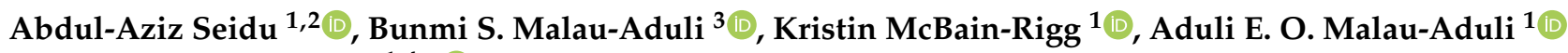 \\ and Theophilus I. Emeto 1,4,*(D) \\ 1 College of Public Health, Medical and Veterinary Sciences, James Cook University, \\ Townsville, QLD 4811, Australia; abdulaziz.seidu@my.jcu.edu.au (A.-A.S.); kris.mcbain@jcu.edu.au (K.M.-R.); \\ aduli.malauaduli@jcu.edu.au (A.E.O.M.-A.) \\ 2 Department of Population and Health, University of Cape Coast, Cape Coast TF 0494, Ghana \\ 3 College of Medicine and Dentistry, James Cook University, Townsville, QLD 4811, Australia; \\ bunmi.malauaduli@jcu.edu.au \\ 4 World Health Organization Collaborating Center for Vector-Borne and Neglected Tropical Diseases, \\ James Cook University, Townsville, QLD 4811, Australia \\ * Correspondence: theophilus.emeto@jcu.edu.au; Tel.: +61-7-4781-5082
}

check for updates

Citation: Seidu, A.-A.; Malau-Aduli, B.S.; McBain-Rigg, K.; Malau-Aduli, A.E.O.; Emeto, T.I. Level of Inclusiveness of People with Disabilities in Ghanaian Health Policies and Reports: A Scoping Review. Disabilities 2021, 1, 257-277. https://doi.org/10.3390/

disabilities1030019

Academic Editor: Nathan Wilson

Received: 26 July 2021

Accepted: 24 August 2021

Published: 29 August 2021

Publisher's Note: MDPI stays neutral with regard to jurisdictional claims in published maps and institutional affiliations.

Copyright: (C) 2021 by the authors. Licensee MDPI, Basel, Switzerland. This article is an open access article distributed under the terms and conditions of the Creative Commons Attribution (CC BY) license (https:/ / creativecommons.org/licenses/by/ $4.0 /)$.
Abstract: (1) Background: Globally, persons with disabilities (PWDs) face numerous challenges including access to healthcare. This scoping review sought to assess the level of inclusiveness in Ghanaian health policies and reports for PWDs; (2) Methods: An extensive search and scoping review of health policies/reports in Ghanaian government websites and Google was conducted. The Preferred Reporting Items for Systematic Reviews and Meta-Analyses (PRISMA) guidelines for scoping reviews were followed in selecting and reporting the policies/reports. The program evaluation and policy design framework was employed for content analysis; (3) Results: Fifteen policies and reports with political recognition, objectives, and specific resources needed to execute stipulated plans were included. Although these policies and reports had collaborative partnerships with various institutions and organisations in development and implementation plans, the level of inclusiveness of disability issues was very low. Only eight out of the fifteen policies/reports (53\%) reviewed considered PWDs, indicating a low level of inclusion. The definition of disability was also limited.; (4) Conclusions: To achieve the sustainable development goal of 'leaving no one behind' in line with the provisions of the United Nations Convention on the Right of Persons with Disabilities and the Disability Act 715 of Ghana, it is recommended that subsequent health policies should have an in-depth engagement with PWDs from the conceptual design stage right through to implementation and evaluation.

Keywords: disability; Ghana; policies; scoping review

\section{Introduction}

Out of approximately one billion individuals worldwide with various types of disabilities, 190 million face substantial challenges in carrying out their regular tasks [1-3]. Thus, persons with disabilities (PWDs) are defined as "those who have long-term physical, mental, intellectual, or sensory impairments which in interaction with various barriers, may hinder their full and effective participation in society on an equal basis with others" [4] (p. 4). The number of PWDS is rising, partly due to the ageing population and the rise in chronic health conditions [3]. The prevalence of disability in low- and middle-income countries (LMICs) is higher than in high-income nations and was predicted to be between $10 \%$ and $15 \%$ of the total population (400 million people) in LMICs [1,2]. Eighty million of these people live in Africa. In Ghana, PWDs account for three percent $(3.7 \%)$ of the population [5].

PWDs are a group of people with special needs and are confronted daily with several challenges that transcend different aspects of their lives. World-wide policies and strategies 
to minimize these challenges have been initiated. One of such global initiatives is the United Nations Conventions on the Right of Persons with Disabilities (UNCRPWD) that has been ratified by 182 countries [6], WHO Global Disability Action Plan 2014-2021 to remove barriers and improve access to health services and programmes for PWDs [7], the United Nations Sustainable Development Goals (SDG) 10 outline the commitment to 'Leave no-one behind' [8] in the contextual framework of specific programs and policies aimed at eliminating the challenges confronting PWDs, while Goal 3 seeks to 'Ensure healthy lives and promote wellbeing for all at all ages'. In Ghana for example, the Disability Act 715 was passed in 2006 to improve the well-being of PWDs and to ensure their inclusion in every sphere of society [9].

Despite the widespread ratifications of UNCRPWD goals such as easy accessibility, improvement in health including Sexual and Reproductive Health (SRH), freedom from exploitation, violence and abuse $[10,11]$, PWDs still face several challenges in life; including access to health services [12]. The WHO [7] reports that PWDs have about a three-fold likelihood to be denied medical care and four-times increased likelihood to receive substandard care from health professionals. These challenges may be due to low income, low levels of education, fewer economic opportunities, or inadequate knowledge of healthcare workers about how to interact with PWDs and poor infrastructure. In terms of sexual health, there is a misconception that the sexual health of PWDs is less important than other health priorities $[12,13]$, and the SRH problems further exacerbate these difficulties. The aforementioned challenges are interconnected, showing similarity across settings [14,15], and can be generally categorised into physical structure, transportation [16,17], finance [18], communication and stigmatization [19]. As a result of these challenges, PWDs have relatively high exposure to adverse health outcomes [20] such as poor health, low quality of life, chronicity of preventable health conditions, comorbidities, additional impairments, and SRH-related outcomes including unintended pregnancies [21], miscarriages [10,11], preterm and low birth weight babies, sexually transmitted infections (STIs) including HIV and AIDs, sexual abuse, and unsafe abortions [22,23]. The overall health of PWDs, including their SRH, is important because, when these health problems are not tackled, they place a huge economic burden on PWDs, their families, society, and the nation at large [14]. PWDs need to spend their limited resources seeking healthcare. PWDs who are not employed, in most cases, rely on their families or caregivers for resources to seek healthcare. The nation, as a whole, also spends additional resources to ensure that those health problems are solved [14].

The 1994 Cairo International Conference on Population and Development (ICPD) policy framework has shaped health policies and SRH interventions across various populations and has been the driving force behind Ghana's considerable SRH achievements [24,25]. This is evident in the numerous health policies that have emerged over the years including the National Health Policy (NHP), Reproductive Health Strategic Plan (RHSP) 2007-2011, National HIV / AIDS and STI Policy, and the Ghana Adolescent Health Service Policy and Strategy (AHSPS) 2016-2020 [24,26,27].

The population of PWDs in Ghana is high, but they are still under-represented in numerous health policies. Within Africa, Lang et al. [28] in their analysis of 7 policies of the African Union, revealed that PWDs are not well incorporated in all the policies. There were problems with target definition, monitoring and evaluation of activities outlined by the policies. In Ghana, Mprah et al. [29] revealed that the National Population Policy (NPP) categorises PWDs and the aged as a homogenous group with similar prescribed strategies, even though they have distinct health needs. Other policies, such as the Ghana National HIV / AIDS \& STI Policy, also discuss disability in the context of social protection but not within the frame of SRH [30]. Generally, the perception among population of persons without disability is that PWDs are not sexually active, therefore, there is no need to advocate and make provisions for them in some health policies, and in SRH policies in particular [31]. Nonetheless, SRH is a major component of health and a greater resource for sustainable development but risky sexual practices, unsafe abortions, STIs and other 
pregnancy-related complications greatly contribute to the morbidity and mortality in many LMICs [29,30,32]. Despite this, the SRH needs of PWDs have been neglected [29,30,32]. According to Hashemi et al. [32], PWDs are more likely to have poor general health outcomes when compared to those without disabilities. Therefore, the omission of disability in health policies in Ghana may pose a threat to their needs and outcomes [31]. For example, Jamali [33] postulates that less priority and specificity of disability in SRH policies can significantly reduce the likelihood for PWDs to seek HIV counselling and related health services. The non-inclusion of disability in health policies may retard advocacy and health interventions targeted at PWDs since there are no clear guidelines and implementation targets. Moreover, the marginalization and stigmatization of PWDs based on their SRH choices and needs may be potentially compounded if efforts are not made to foster their inclusion in Ghanaian health policies. This is reiterated by Mfoafo-M'Carthy et al. [31] who recommended that, in order to advance the rights of PWDs in Ghana, a move towards inclusiveness should be considered.

Previous studies on the inclusion of disability in policies and research have been conducted by Mprah et al. [29] and Abdul Karimu [30]. These reviews were specific and limited in scope. Specifically, Mprah et al. [29] reviewed how disability-related issues have been included in SRH policies and research, while Abdul Karimu [30] examined the inclusion of adolescents with disabilities in the AHSPS 2016-2020. This current review is novel as it considers all the major health policies and reports in general. It also uses the most recent policies in health to see if there has been a change or addition as far as disability and health policies/reports are concerned in Ghana. Therefore, to promote inclusiveness of disability in health policies/reports in Ghana, it is important to understand the current level of inclusion of PWDs. The primary aim of this review, therefore, is to present a comprehensive analysis of the level of inclusiveness of disability in Ghanaian health policies/reports. With SDG 3 and 10 aiming to 'ensure healthy lives and promote wellbeing for all at all ages' and to 'leave no one behind', respectively, this review is relevant in promoting equality, dignity and nondiscrimination in the scope of health policies in Ghana. As Ghana aims to achieve universal health coverage, this review is timely because it will inform policy and implementation strategies towards inclusiveness in the country's health policies. It will also serve as a progress barometer for gauging the objective of achieving inclusiveness in the disability act of Ghana since its inception in 2006.

\section{Materials and Methods}

For this scoping review, the authors followed the five major steps of the methodological framework of Arksey and O'Malley [34]: (1) "identifying the review/research question" (2) "identifying relevant studies (policies/documents)", (3) "study selection", (4) "charting the data", and (5) "collating, summarizing, and reporting the results". We skipped the framework's consultation phase because this is an optional step. Previous studies in sub-Saharan Africa, notably in Ghana [25] and elsewhere [35], used this methodological framework in the scoping review of policies.

\subsection{Phase 1: The Review/Research Question}

The following review questions were considered:

(1) What are the health policies/reports in Ghana?

(2) To what extent do these policies/reports include PWDs?

Another conceptual framework for evaluating program and policy design on adolescent reproductive health was utilised to define the review objectives and guide data analysis [25,36] (Figure 1). This framework was chosen for two reasons: First, it has been applied in previous SSA studies [25,36] including Ghana [25]. Second, some of the framework's core issues are key extractable variables from the policies to assess the extent of disability inclusion in Ghana's health policies/reports. 


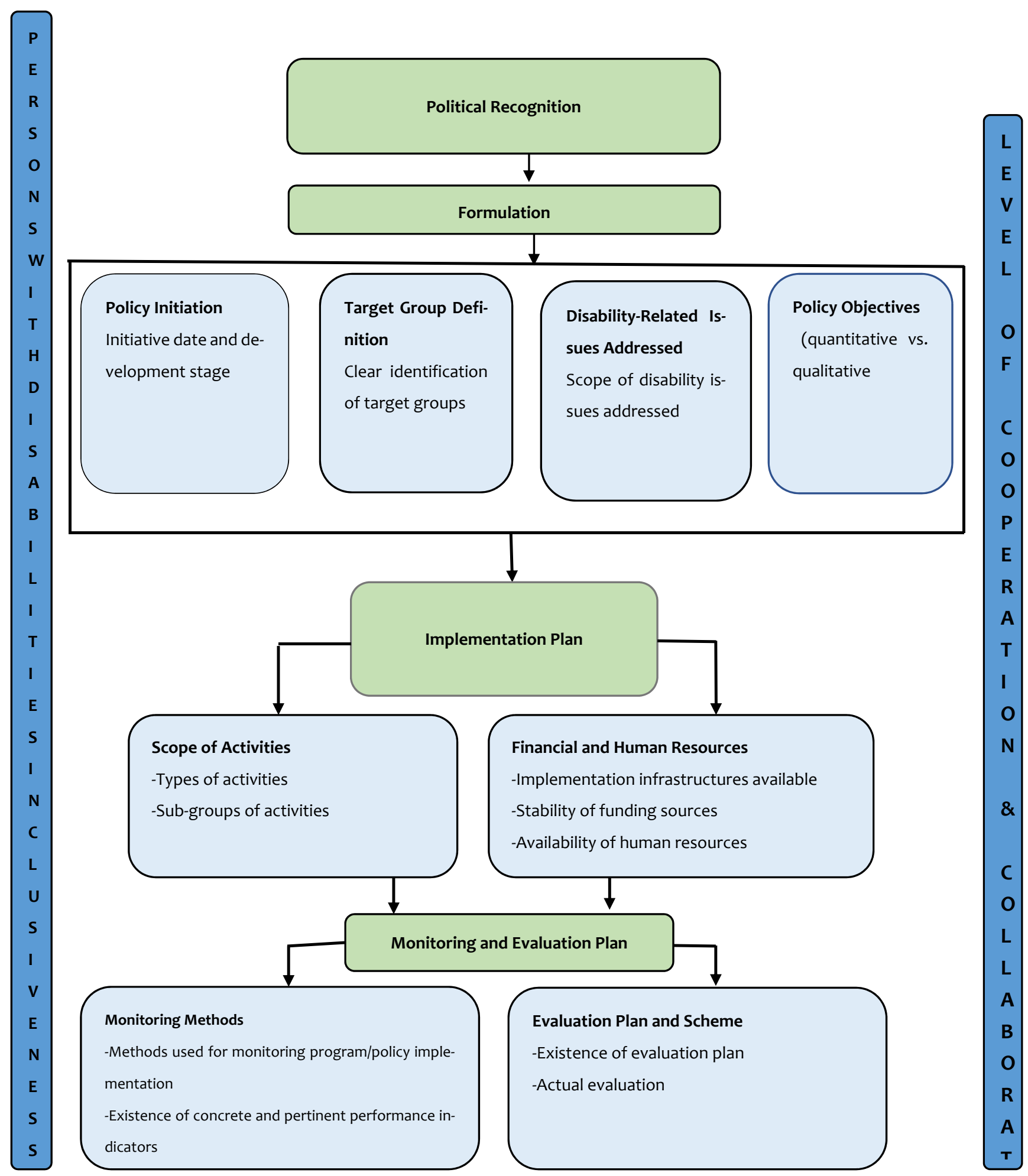

Figure 1. Reproduced with permission: Conceptual framework for evaluating program and policy design. Source: Calves [36].

The various components of the framework and its application in this review are as follows: The degree to which disability-related issues are recognised as national issues and are prioritized is referred to as political recognition. This could be in the form of the government officials or, in some cases, the relevant minister (designated government 
official) signing a particular policy. It could also take the shape of political leaders' statements expressing their support for the goals and objectives of the policy. In this sense, the inclusion of disability-related concerns such as access to health care, stigma, discrimination and economic empowerment within the country. The formulation component considers the policy's inception date and stage of development, and whether the policy is new or an amended version. The people for whom the policy was established are referred to as the target group. The scope of operations and financial and human resources used to implement the policy are all considered in the implementation plan of the policy. The monitoring and evaluation plan focuses on monitoring methods and the inclusion of an assessment plan in the policy to ensure that the objectives are achieved. The roles and number of partners involved in the policy are assessed in terms of coordination and collaboration. Finally, the extent and level of inclusion is viewed as how the target groups (PWDs) are involved at each level of the policy formulation process [25,36] (See Figure 1).

\subsection{Phase 2: Identifying Relevant Policies}

The data were gathered from Ghanaian health policies/reports. The main criterion for inclusion was being a health policy/report. There was no limit on the age of the policy, however, with policies that were revised, the most recent version was used. An initial search was conducted using the following databases: Scopus, Medline/PubMed, CINAHL/EBSCO, and Web of Science. Search terms employed included "sexual and reproductive health policies" or "health promotion policies" or "health education policies" or "health services policies" or "youth policies" or "reproductive health policy" or "sexual health policy" or "sexual education" or "maternal health policy" or "health insurance policy" or "contraception or abortion" or "sexual health or STIs or STDs policy" or "comprehensive sexuality education policy" or "family planning and contraception or sexual violence or sexuality policy". This search yielded only one result [29]. As a result, we resorted to Google to search for health policies/reports via the websites of national health departments/ministries. The search took place between 17th May and 24th May 2021. The various websites of the ministries such as the Ministry of Health (MOH) (https:/ /www.moh.gov.gh/policy-documents/, accessed on 18 May 2021), Ministry of Gender, Children and Social Protection (MGCSP) (https:/ /www.mogcsp.gov.gh/, accessed on 18 May 2021), National Population Council, Ghana Statistical Service (https: / /statsghana.gov.gh/, accessed on 20 May 2021) and the Ministry of Education (MOE) (https:/ / moe.gov.gh, accessed on 20 May 2021) were searched. The search found 41 policies/reports from Google and 23 policies/reports on government websites.

\subsection{Phase 3: Study Selection}

As shown in the PRISMA flow diagram in Figure 2, a screening procedure was used to select the final sample of policies and reports for review [37] (Supplementary Table S1). Of the 64 policies/reports retrieved from phase 1, 30 were excluded because they did not meet the eligibility criteria. Nine policies/reports were excluded because they were old versions of otherwise included documents. In the final stage, 15 policies/reports were included in this scoping review. These were the NHP [38], the 1994 NPP [39], National HIV and AIDS, STI policy [27], Adolescent Health Service Policy and Strategy (AHSPS) [40], the 2007 Reproductive health strategic plan (RHSP) [41], the 2016 Domestic Violence Report (DVR) [42], the Criminal Offence Code (COC) [43], the National Survey of Adolescents (NSA) [44], the National Health Insurance Scheme policy (NHISP) [45], National Gender Policy (NGP) [46], the 2017/18 Multiple indicator cluster survey (MICS) [47], National Reproductive health service policy and standards (NRHPS) [48], the Ghana Family Planning Costed Implementation Plan (GFPCIP) [49], the 2014 Ghana Demographic and Health Survey (GDHS) [50] and the 2017 Ghana Maternal Health Survey (GMHS) [51]. Figure 2 depicts the policy search and screening process in detail. 


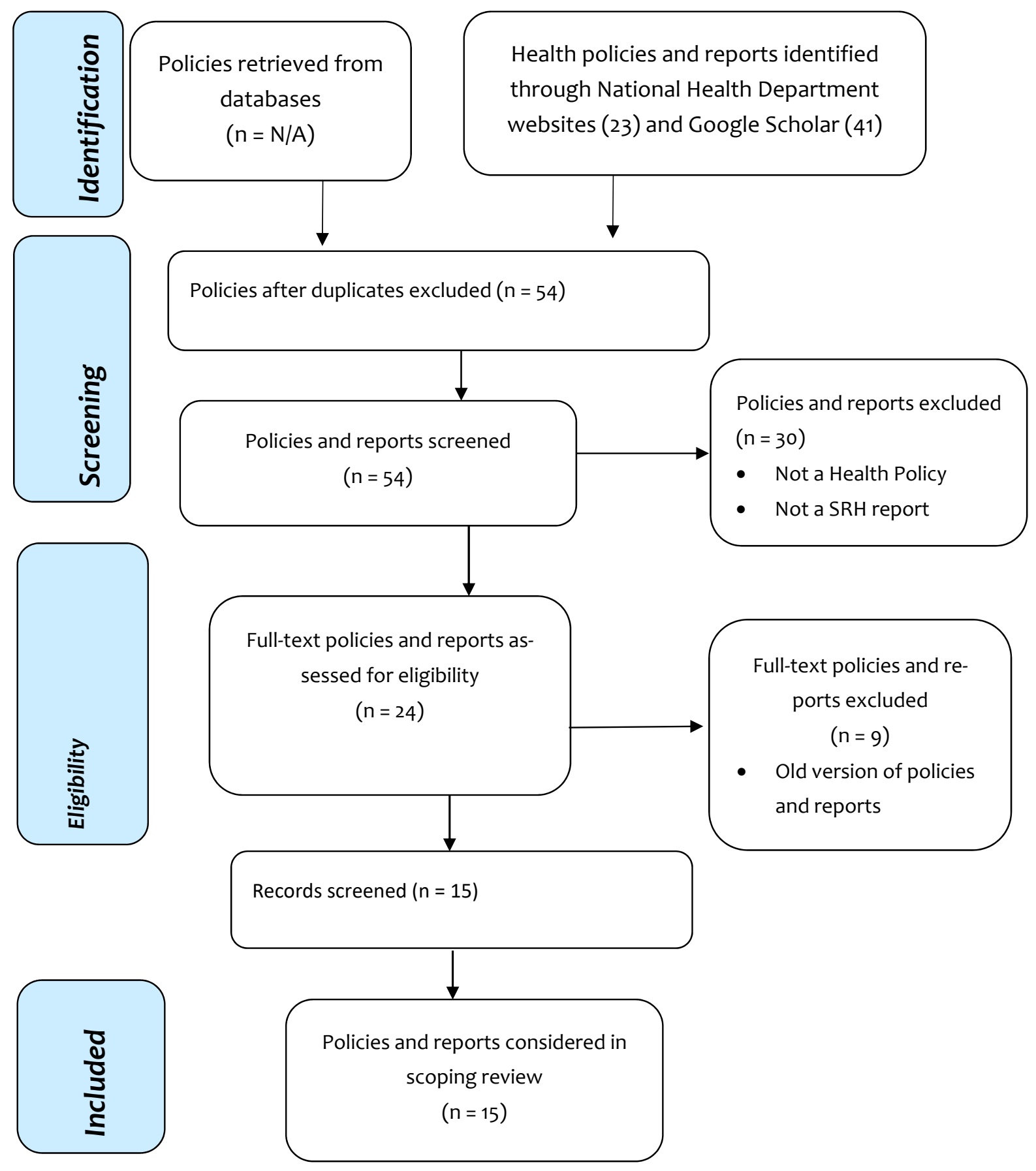

Figure 2. PRISMA extension for scoping reviews (PRISMA-ScR) diagram of included policies and reports [37].

\subsection{Phase 4: Charting the Data}

A matrix was constructed in Excel for extracting and displaying data from the policies (Table 1). The matrix aided data organization by ensuring that extracted data aligned with the components of the conceptual framework (Figure 1). The specific variables of interest guided by the framework included: (1) "political recognition", (2) "policy initiation", (3) "target group definition", (4) "policy objectives", (5) "scope of activities", (6) "financial and human resources", (7) "monitoring and evaluation plan" (8) "level of cooperation and collaboration", and (10) "level of PWDs inclusiveness in health policies/reports in Ghana". 
Table 1. Summary of health Policies/Reports included in the review.

\begin{tabular}{|c|c|c|c|c|c|c|c|c|c|c|c|c|c|}
\hline $\begin{array}{c}\text { Policy/Report } \\
\text { Title }\end{array}$ & $\begin{array}{c}\text { Political } \\
\text { Recognition }\end{array}$ & $\begin{array}{l}\text { Policy/ } \\
\text { Report } \\
\text { Initiation }\end{array}$ & $\begin{array}{c}\text { Disability/Issues } \\
\text { Addressed } \\
\text { Concerning } \\
\text { Persons with } \\
\text { Disabilities }\end{array}$ & $\begin{array}{l}\text { Target Group } \\
\text { Definition }\end{array}$ & $\begin{array}{c}\text { Composition } \\
\text { of the People } \\
\text { who } \\
\text { Developed It/ } \\
\text { Advisory } \\
\text { Panel }\end{array}$ & $\begin{array}{c}\text { Policy } \\
\text { Objective } \\
\text { (Qualitative/ } \\
\text { Quantitative) }\end{array}$ & $\begin{array}{l}\text { Scope of } \\
\text { Activities/ } \\
\text { Strategies to } \\
\text { Include } \\
\text { PWDs }\end{array}$ & $\begin{array}{l}\text { Resources } \\
\text { (Financial } \\
\text { and Human) }\end{array}$ & $\begin{array}{l}M \text { and E Plan } \\
\text { and } \\
\text { Performance } \\
\text { Indicators }\end{array}$ & $\begin{array}{c}\text { Cooperation } \\
\text { \& Collabora- } \\
\text { tion } \\
\text { (Number of } \\
\text { Partners } \\
\text { Involved) }\end{array}$ & $\begin{array}{c}\text { Involvement } \\
\text { of Persons } \\
\text { with } \\
\text { Disabilities }\end{array}$ & $\begin{array}{c}\text { How } \\
\text { Accessible Is } \\
\text { It? }\end{array}$ & $\begin{array}{c}\text { Mention } \\
\text { of Dis- } \\
\text { ability }\end{array}$ \\
\hline $\begin{array}{l}\text { National } \\
\text { Health Policy: } \\
\text { Ensuring } \\
\text { healthy lives } \\
\text { for all, } \\
\text { revised } \\
\text { version, } \\
\text { January 2020 } \\
\text { [38] } \\
\end{array}$ & yes & $01 / 01 / 2020$ & $\begin{array}{c}\text { access to } \\
\text { healthcare, } \\
\text { discrimination } \\
\text { and stigmatisation } \\
\text { and } \\
\text { empowerment }\end{array}$ & general & $\begin{array}{l}\text { multi- } \\
\text { disciplinary } \\
\text { group, no } \\
\text { disability } \\
\text { organisation } \\
\text { present }\end{array}$ & qualitative & $x$ & $\begin{array}{l}\text { human and } \\
\text { financial } \\
\text { resources }\end{array}$ & $\sqrt{ }$ & $\sqrt{ }$ & $\checkmark$ & $\begin{array}{l}\text { online } \\
\text { (English } \\
\text { version) }\end{array}$ & yes \\
\hline $\begin{array}{c}\text { Ghana } \\
\text { Demographic } \\
\text { and Health } \\
\text { Survey (2014) } \\
\text { [50] }\end{array}$ & yes & 2014 & $x$ & $\begin{array}{l}\text { men (15-64), } \\
\text { women } \\
\text { (15-49), } \\
\text { children } \\
\text { (under five) }\end{array}$ & $\begin{array}{c}\text { multi- } \\
\text { disciplinary } \\
\text { group, no } \\
\text { disability } \\
\text { organisation } \\
\text { present } \\
\end{array}$ & qualitative & $\mathrm{x}$ & $\begin{array}{l}\text { human and } \\
\text { financial } \\
\text { resources }\end{array}$ & N/A & $\checkmark$ & $x$ & $\begin{array}{l}\text { online } \\
\text { (English } \\
\text { version) }\end{array}$ & yes \\
\hline $\begin{array}{c}\text { Ghana } \\
\text { Maternal } \\
\text { Health } \\
\text { Survey(2017) } \\
{[51]}\end{array}$ & yes & 2017 & $\begin{array}{c}\text { health care access, } \\
\text { insurance, and } \\
\text { disability, family } \\
\text { planning, ANC, } \\
\text { skilled delivery, } \\
\text { place of delivery, } \\
\text { problems in } \\
\text { accessing } \\
\text { healthcare }\end{array}$ & $\begin{array}{c}\text { women aged } \\
15-49\end{array}$ & $\begin{array}{l}\text { multi- } \\
\text { disciplinary } \\
\text { group, no } \\
\text { disability } \\
\text { organisation } \\
\text { present }\end{array}$ & qualitative & $x$ & $\begin{array}{l}\text { human and } \\
\text { financial } \\
\text { resources }\end{array}$ & N/A & $\checkmark$ & $x$ & $\begin{array}{l}\text { online } \\
\text { (English } \\
\text { version) }\end{array}$ & yes \\
\hline $\begin{array}{c}\text { National } \\
\text { Population } \\
\text { Policy [39] }\end{array}$ & yes & 1994 & $\begin{array}{c}\text { creating } \\
\text { opportunities for } \\
\text { participation. }\end{array}$ & general & $\begin{array}{l}\text { multi- } \\
\text { disciplinary, } \\
\text { no disability } \\
\text { organisation } \\
\text { present }\end{array}$ & qualitative & $\begin{array}{l}\text { Education, } \\
\text { form a } \\
\text { national } \\
\text { coordinating } \\
\text { committee on } \\
\text { disability, } \\
\text { enactment of } \\
\text { laws and } \\
\text { enforcement } \\
\text { of existing } \\
\text { ones, } \\
\text { provision of } \\
\text { assistance }\end{array}$ & $\begin{array}{l}\text { human and } \\
\text { financial } \\
\text { resources }\end{array}$ & $\checkmark$ & $\checkmark$ & $x$ & $\begin{array}{l}\text { online } \\
\text { (English } \\
\text { version) }\end{array}$ & yes \\
\hline $\begin{array}{l}\text { National HIV } \\
\text { and AIDS, } \\
\text { STI policy } \\
\text { [27] }\end{array}$ & yes & $01 / 02 / 2013$ & $x$ & $\begin{array}{l}\text { general } \\
\text { population } \\
\text { with much } \\
\text { emphasis on } \\
\text { persons } \\
\text { living with } \\
\text { HIV and } \\
\text { AIDS }\end{array}$ & $\begin{array}{l}\text { multi- } \\
\text { disciplinary } \\
\text { group, no } \\
\text { disability } \\
\text { organisation } \\
\text { present }\end{array}$ & qualitative & $\begin{array}{l}\text { IEC (social } \\
\text { and } \\
\text { behavioural } \\
\text { change, } \\
\text { including } \\
\text { most at risk } \\
\text { populations. } \\
\text { Social } \\
\text { protection }\end{array}$ & $\begin{array}{l}\text { human and } \\
\text { financial } \\
\text { resources }\end{array}$ & $\checkmark$ & $\sqrt{ }$ & $x$ & $\begin{array}{l}\text { online } \\
\text { (English } \\
\text { version) }\end{array}$ & yes \\
\hline
\end{tabular}


Table 1. Cont

\begin{tabular}{|c|c|c|c|c|c|c|c|c|c|c|c|c|c|}
\hline $\begin{array}{c}\text { Policy/Report } \\
\text { Title }\end{array}$ & $\begin{array}{c}\text { Political } \\
\text { Recognition }\end{array}$ & $\begin{array}{l}\text { Policy/ } \\
\text { Report } \\
\text { Initiation }\end{array}$ & $\begin{array}{l}\text { Disability/Issues } \\
\text { Addressed } \\
\text { Concerning } \\
\text { Persons with } \\
\text { Disabilities }\end{array}$ & $\begin{array}{l}\text { Target Group } \\
\text { Definition }\end{array}$ & $\begin{array}{c}\text { Composition } \\
\text { of the People } \\
\text { who } \\
\text { Developed It/ } \\
\text { Advisory } \\
\text { Panel }\end{array}$ & $\begin{array}{c}\text { Policy } \\
\text { Objective } \\
\text { (Qualitative/ } \\
\text { Quantitative) }\end{array}$ & $\begin{array}{l}\text { Scope of } \\
\text { Activities/ } \\
\text { Strategies to } \\
\text { Include } \\
\text { PWDs }\end{array}$ & $\begin{array}{l}\text { Resources } \\
\text { (Financial } \\
\text { and Human) }\end{array}$ & $\begin{array}{l}M \text { and E Plan } \\
\text { and } \\
\text { Performance } \\
\text { Indicators }\end{array}$ & $\begin{array}{c}\text { Cooperation } \\
\text { \& Collabora- } \\
\text { tion } \\
\text { (Number of } \\
\text { Partners } \\
\text { Involved) }\end{array}$ & $\begin{array}{l}\text { Involvement } \\
\text { of Persons } \\
\text { with } \\
\text { Disabilities }\end{array}$ & $\begin{array}{c}\text { How } \\
\text { Accessible Is } \\
\text { It? }\end{array}$ & $\begin{array}{c}\text { Mention } \\
\text { of Dis- } \\
\text { ability }\end{array}$ \\
\hline $\begin{array}{l}\text { Reproductive } \\
\text { health } \\
\text { strategic plan } \\
\text { [41] }\end{array}$ & yes & $07 / 04 / 2007$ & $x$ & general & $\begin{array}{l}\text { multi- } \\
\text { disciplinary } \\
\text { group, no } \\
\text { disability } \\
\text { organisation } \\
\text { present }\end{array}$ & qualitative & $\begin{array}{c}\text { IEC } \\
\text { (Information, } \\
\text { education } \\
\text { and commu- } \\
\text { nication, } \\
\text { Behavioural } \\
\text { Change Com- } \\
\text { munication), } \\
\text { improving } \\
\text { access to } \\
\text { MHS (ANC, } \\
\text { Skilled } \\
\text { delivery, } \\
\text { PNC), } \\
\text { Promotion of } \\
\text { access to } \\
\text { contraceptive } \\
\text { prevalence, } \\
\text { using } \\
\text { community } \\
\text { based } \\
\text { volunteers }\end{array}$ & $\begin{array}{l}\text { human and } \\
\text { financial } \\
\text { resources }\end{array}$ & $\checkmark$ & $\sqrt{ }$ & $x$ & $\begin{array}{l}\text { online } \\
\text { (English } \\
\text { version) }\end{array}$ & no \\
\hline $\begin{array}{c}\text { Domestic } \\
\text { Violence } \\
\text { Report [42] }\end{array}$ & yes & $01 / 07 / 2016$ & $x$ & $\begin{array}{l}\text { men, women, } \\
\text { boys and } \\
\text { girls between } \\
\text { the ages of } 15 \\
\text { and } 60 \text { years }\end{array}$ & $\begin{array}{c}\text { multi- } \\
\text { disciplinary } \\
\text { group, no } \\
\text { disability } \\
\text { organisation } \\
\text { present }\end{array}$ & qualitative & $x$ & $\begin{array}{l}\text { human and } \\
\text { financial } \\
\text { resources }\end{array}$ & N/A & $\sqrt{ }$ & $x$ & $\begin{array}{l}\text { online } \\
\text { (English } \\
\text { version) }\end{array}$ & yes \\
\hline
\end{tabular}


Table 1. Cont.

\begin{tabular}{|c|c|c|c|c|c|c|c|c|c|c|c|c|c|}
\hline $\begin{array}{l}\text { Policy/Report } \\
\text { Title }\end{array}$ & $\begin{array}{c}\text { Political } \\
\text { Recognition }\end{array}$ & $\begin{array}{l}\text { Policy/ } \\
\text { Report } \\
\text { Initiation }\end{array}$ & $\begin{array}{l}\text { Disability/Issues } \\
\text { Addressed } \\
\text { Concerning } \\
\text { Persons with } \\
\text { Disabilities }\end{array}$ & $\begin{array}{l}\text { Target Group } \\
\text { Definition }\end{array}$ & $\begin{array}{c}\text { Composition } \\
\text { of the People } \\
\text { who } \\
\text { Developed It/ } \\
\text { Advisory } \\
\text { Panel }\end{array}$ & $\begin{array}{c}\text { Policy } \\
\text { Objective } \\
\text { (Qualitative/ } \\
\text { Quantitative) }\end{array}$ & $\begin{array}{l}\text { Scope of } \\
\text { Activities/ } \\
\text { Strategies to } \\
\text { Include } \\
\text { PWDs }\end{array}$ & $\begin{array}{l}\text { Resources } \\
\text { (Financial } \\
\text { and Human) }\end{array}$ & $\begin{array}{c}M \text { and E Plan } \\
\text { and } \\
\text { Performance } \\
\text { Indicators }\end{array}$ & $\begin{array}{l}\text { Cooperation } \\
\text { \& Collabora- } \\
\text { tion } \\
\text { (Number of } \\
\text { Partners } \\
\text { Involved) }\end{array}$ & $\begin{array}{c}\text { Involvement } \\
\text { of Persons } \\
\text { with } \\
\text { Disabilities }\end{array}$ & $\begin{array}{c}\text { How } \\
\text { Accessible Is } \\
\text { It? }\end{array}$ & $\begin{array}{c}\text { Mention } \\
\text { of Dis- } \\
\text { ability }\end{array}$ \\
\hline $\begin{array}{c}\text { Criminal } \\
\text { offences code } \\
{[43]}\end{array}$ & yes & 12/01/1961 & abortion & general & not stated & qualitative & $x$ & $\begin{array}{l}\text { human and } \\
\text { financial } \\
\text { resources }\end{array}$ & N/A & $x$ & $x$ & $\begin{array}{l}\text { online } \\
\text { (English } \\
\text { version) }\end{array}$ & yes \\
\hline $\begin{array}{c}\text { National } \\
\text { Survey of } \\
\text { Adolescents } \\
\text { [44] }\end{array}$ & not described & $01 / 06 / 2004$ & $x$ & $\begin{array}{l}\text { adolescents } \\
\text { (10-19 years) }\end{array}$ & $\begin{array}{l}\text { group of } \\
\text { researchers } \\
\text { without } \\
\text { inputs from } \\
\text { persons with } \\
\text { disabilities }\end{array}$ & qualitative & $x$ & $\begin{array}{l}\text { human and } \\
\text { financial } \\
\text { resources }\end{array}$ & N/A & $\mathrm{x}$ & $x$ & $\begin{array}{l}\text { online } \\
\text { (English } \\
\text { version) }\end{array}$ & no \\
\hline $\begin{array}{l}\text { National } \\
\text { Health } \\
\text { Insurance } \\
\text { Scheme } \\
\text { Policy [45] }\end{array}$ & yes & August, 2004 & $\begin{array}{l}\text { exemption from } \\
\text { payment }\end{array}$ & general & $\begin{array}{c}\text { multi- } \\
\text { disciplinary } \\
\text { group, no } \\
\text { disability } \\
\text { organisation } \\
\text { present } \\
\end{array}$ & qualitative & $\begin{array}{c}\text { Exemptions } \\
\text { of payment } \\
\text { for Poor and } \\
\text { Vulnerable } \\
\text { groups }\end{array}$ & $\begin{array}{l}\text { human and } \\
\text { financial } \\
\text { resources }\end{array}$ & $x$ & $\sqrt{ }$ & $\mathrm{x}$ & $\begin{array}{l}\text { online } \\
\text { (English } \\
\text { version) }\end{array}$ & yes \\
\hline $\begin{array}{l}\text { National } \\
\text { Gender } \\
\text { Policy [46] }\end{array}$ & yes & May, 2015 & $\begin{array}{l}\text { opportunities for } \\
\text { participation }\end{array}$ & general & $\begin{array}{l}\text { multi- } \\
\text { disciplinary } \\
\text { group, no } \\
\text { disability } \\
\text { organisation } \\
\text { present }\end{array}$ & qualitative & $\begin{array}{l}\text { Information } \\
\text { Education } \\
\text { and Commu- } \\
\text { nication } \\
\text { (Social and } \\
\text { Behavioural } \\
\text { Change Com- } \\
\text { munication } \\
\text { strategy), } \\
\text { Health } \\
\text { Services, } \\
\text { Training } \\
\text { (needs } \\
\text { assessment of } \\
\text { staff, and } \\
\text { capacity } \\
\text { building of } \\
\text { staff) }\end{array}$ & $\begin{array}{l}\text { human and } \\
\text { financial } \\
\text { resources }\end{array}$ & $\sqrt{ }$ & $\sqrt{ }$ & $\sqrt{ }$ & $\begin{array}{l}\text { online } \\
\text { (English } \\
\text { version) }\end{array}$ & yes \\
\hline $\begin{array}{c}\text { Multiple } \\
\text { indicator } \\
\text { cluster } \\
\text { survey [47] }\end{array}$ & yes & 2018 & $\begin{array}{l}\text { functioning based } \\
\text { on the WHO } \\
\text { functional } \\
\text { disability mod- } \\
\text { ule/questionnaire }\end{array}$ & $\begin{array}{c}\text { men and } \\
\text { women, } \\
\text { children aged } \\
5-17 \text { years as } \\
\text { well as } \\
\text { children } \\
\text { under } 5\end{array}$ & $\begin{array}{l}\text { not clearly } \\
\text { stated }\end{array}$ & qualitative & $\begin{array}{l}\text { Adding the } \\
\text { functional } \\
\text { disability } \\
\text { module to the } \\
\text { survey }\end{array}$ & $\begin{array}{l}\text { human and } \\
\text { financial } \\
\text { resources }\end{array}$ & N/A & $\sqrt{ }$ & Yes & $\begin{array}{l}\text { online } \\
\text { (English } \\
\text { version) }\end{array}$ & yes \\
\hline
\end{tabular}


Table 1. Cont.

\begin{tabular}{|c|c|c|c|c|c|c|c|c|c|c|c|c|c|}
\hline $\begin{array}{l}\text { Policy/Report } \\
\text { Title }\end{array}$ & $\begin{array}{c}\text { Political } \\
\text { Recognition }\end{array}$ & $\begin{array}{c}\text { Policy/ } \\
\text { Report } \\
\text { Initiation }\end{array}$ & $\begin{array}{c}\text { Disability/Issues } \\
\text { Addressed } \\
\text { Concerning } \\
\text { Persons with } \\
\text { Disabilities }\end{array}$ & $\begin{array}{c}\text { Target Group } \\
\text { Definition }\end{array}$ & $\begin{array}{c}\text { Composition } \\
\text { of the People } \\
\text { who } \\
\text { Developed It/ } \\
\text { Advisory } \\
\text { Panel }\end{array}$ & $\begin{array}{c}\text { Policy } \\
\text { Objective } \\
\text { (Qualitative/ } \\
\text { Quantitative) }\end{array}$ & $\begin{array}{l}\text { Scope of } \\
\text { Activities/ } \\
\text { Strategies to } \\
\text { Include } \\
\text { PWDs }\end{array}$ & $\begin{array}{l}\text { Resources } \\
\text { (Financial } \\
\text { and Human) }\end{array}$ & $\begin{array}{c}M \text { and E Plan } \\
\text { and } \\
\text { Performance } \\
\text { Indicators }\end{array}$ & $\begin{array}{l}\text { Cooperation } \\
\text { \& Collabora- } \\
\text { tion } \\
\text { (Number of } \\
\text { Partners } \\
\text { Involved) }\end{array}$ & $\begin{array}{c}\text { Involvement } \\
\text { of Persons } \\
\text { with } \\
\text { Disabilities }\end{array}$ & $\begin{array}{c}\text { How } \\
\text { Accessible Is } \\
\text { It? }\end{array}$ & $\begin{array}{c}\text { Mention } \\
\text { of Dis- } \\
\text { ability }\end{array}$ \\
\hline $\begin{array}{c}\text { National } \\
\text { Reproductive } \\
\text { health service } \\
\text { policy and } \\
\text { standards } \\
{[48]}\end{array}$ & yes & 2014 & $\begin{array}{l}\text { abortion and } \\
\text { contraception }\end{array}$ & general & $\begin{array}{l}\text { not clearly } \\
\text { stated }\end{array}$ & qualitative & $\begin{array}{l}\text { Birth control } \\
\text { for "mentally } \\
\text { disabled" } \\
\text { women. }\end{array}$ & $\begin{array}{l}\text { human and } \\
\text { financial } \\
\text { resources }\end{array}$ & $\sqrt{ }$ & $\sqrt{ }$ & Yes & $\begin{array}{l}\text { online } \\
\text { (English } \\
\text { version) }\end{array}$ & yes \\
\hline $\begin{array}{l}\text { Ghana } \\
\text { Family } \\
\text { Planning } \\
\text { Costed Imple- } \\
\text { mentation } \\
\text { Plan [40] }\end{array}$ & yes & $01 / 09 / 2015$ & $x$ & women & $\begin{array}{l}\text { not clearly } \\
\text { stated }\end{array}$ & $\begin{array}{l}\text { qualitative } \\
\text { and } \\
\text { quantitative }\end{array}$ & $x$ & $\begin{array}{l}\text { human and } \\
\text { financial } \\
\text { resources }\end{array}$ & $\sqrt{ }$ & $\sqrt{ }$ & $x$ & $\begin{array}{l}\text { online } \\
\text { (English } \\
\text { version) }\end{array}$ & yes \\
\hline
\end{tabular}

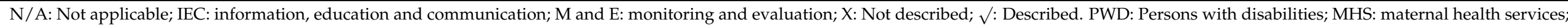
ANC: Antenatal care; PNC: Postnatal care; STIs: Sexually transmitted infections. 


\subsection{Phase 5: Collating, Summarizing and Reporting the Results}

The fifth stage of the methodological framework of Arksey and O'Malley [34] entails collating, summarizing and reporting the results. This was guided by content analysis which involves "a systematic coding and categorizing approach used for exploring large amounts of textual information unobtrusively to determine trends and patterns of words used, their frequency, their relationships, and the structures and discourses of communication" [52]. Content analysis examines who says what, to whom, and with what effect in order to identify the qualities of a document's content [53]. This was used to analyse the policies/reports using the conceptual framework as a guide (Figure 1).

\section{Results}

Table 1 shows the essential components of the health policies/reports based on the conceptual Framework (Figure 1). The specific issues extracted from the policies/reports are also discussed as follows:

\subsection{Political Recognition}

From the 15 policies/reports reviewed, the NSA was the only document that did not show any evidence of political recognition. Political recognition in the 14 documents were indicated by government officials or, in some cases, the relevant minister (designated government official) signing the documents. For example, the NHP [38] was signed by the current President of the Republic of Ghana and the Minister of Health. The 2013 National HIV and AIDS STI policy was also signed by the former President of the Republic of Ghana [27]. All these reflect the political recognition of the policies. Political recognition was also evident in some of the statements made by government and government representatives on some of the documents. A typical example is captured in this excerpt from the AHSPS [40] (p. ii):

"there is the need to create a safe and supportive environment, ensure access to appropriate information, ensure that health facilities provide goods and services including counselling on mental health, nutrition, sexual and reproductive health, ensure the active participation of young people in programmes affecting them and their protection from all forms of violence, harmful practices especially focusing on the more vulnerable such as pre adolescents, younger adolescents, pregnant adolescents, adolescents practising prostitution directly or indirectly and adolescents living with HIV and AIDS".

As most of the policies gained political recognition, hence the need to align them with international goals or targets such as the SDGs. For instance, the current President of the Republic of Ghana made this statement in the NHP [38] (p. 7).

"... The Policy has been inspired by the overall national medium-term policy framework, set out in the Coordinated Programme of Economic and Social Development Policies (2017-2024), and recognises several global, continental and regional compacts and policy frameworks, such as SDGS, the International Health Regulations, the African Union Vision 2063: The Africa We Want, the ECOWAS Vision 2020 and the African Health Strategy (2016-2030)".

\subsection{Policy Initiation}

In terms of policy initiation, the 15 selected policies/reports spanned from the 1960s to 2020. Some of the policies, such as National HIV and AIDS, STI policy [27], NHP [38], and the 1994 NPP [39] were revised policies, while the others were newly implemented policies aimed at monitoring the general population's health and well-being. The amended policies were adopted to keep up with the changing nature of health challenges confronting Ghanaians. For example, the 2013 National HIV and AIDS, STI policy [27] was revised to accommodate the new dynamics of STIs within the country. It emphasised the intensification of HIV prevention efforts by prioritising core groups of people, such as men who have sex with men, female sex workers, people who inject drugs, prisoners, clients of female sex 
workers, and sexual- and needle-sharing partners of persons living with HIV. In the same vein, the NPP was also revised in 1994 to tackle new population dynamics. For instance, the policy was revised to incorporate new conditions such as HIV and AIDS discovered around the 1980s. One of the specific rationales for the policy states: "One other factor that needs careful attention is the issue of sexually transmitted diseases including HIV/AIDS" [39] (p. 7).

\subsection{Target Group Definition}

This review sought to examine the specific groups these policies targeted. While some of the policies targeted specific groups, others targeted the general population. For instance, the 2017 MHS [51] targeted women aged 15-49 years; the AHSPS [40] targeted young people aged 10-24 years, while the National HIV and AIDS, STI policy [27] targeted the general population but with emphasis on people living with HIV and AIDS. While some of the general groups included PWDs [47], others [42] did not. It can be inferred that most of these policies did not consider PWDs as a core group during the policy formulation rocess.

\subsection{Composition of the People Who Developed the Policy/Advisory Panel}

Another key variable considered in this scoping review was the composition of the people who developed the various policies implemented (advisory panel). While most of the policies (e.g., [39]) stated that they consulted several agencies and individuals in drafting the policies, only the AHSPS [40] included the National Commission for PWDs. The remaining 14 policies/reports (NHP, NPP, National HIV and AIDS, STI policy, DVR, AHSPS, COC, NSA, NHISP, NGP, MICS, NRHPS, GFPCIP, GDHAS, and GMHS) did not include any organisation or a person with disability in the design or drafting of the policies/reports. This is another evidence of the low level of inclusion or engagement of PWDs in policy formulation.

\subsection{Disability Issues Addressed}

This section of the review focused on the disability-related issues that were discussed in the various policies/reports. While some of the policies did not include anything related to disability, some of the documents devoted sections to discuss disability-related issues. For example, eight policies (the 2017 GMHS, AHSPS, MICs, the NPP, COC, NHP, NHISP, and the NGP) captured disability-related topics. These included exemption from payment of the National Health Insurance premium, as stipulated in the NHISP [45], the COC [43] also tackled disability-related issues, although the words used to describe PWDs were derogatory. For example, in relation to abortion, one policy states "where the pregnancy is the result of rape, defilement of a female idiot or incest and the abortion or miscarriage is requested by the victim or her next of kin or the person in loco parentis, if she lacks the capacity to make such request" [43] (p. 47).

This is quite problematic (discriminatory) if abortion can be requested by another person on her behalf assuming that she lacks capacity, which is a clear violation of article 12.2 of the UNCRPWD which states that:

"... States/parties shall ensure that all measures that relate to the exercise of legal capacity provide for appropriate and effective safeguards to prevent abuse in accordance with international human rights law. Such safeguards shall ensure that measures relating to the exercise of legal capacity respect the rights, will and preferences of the person, are free of conflict of interest and undue influence, are proportional and tailored to the person's circumstances, apply for the shortest time possible and are subject to regular review by a competent, independent, and impartial authority or judicial body. The safeguards shall be proportional to the degree to which such measures affect the person's rights and interests".

[4] (p. 10-11). 
In the AHSPS [40] (p. 26) it states that "Special attention shall be given in provision of adolescent and youth health education and services to ensure that the peculiar needs of those with disabilities and vulnerable groups are recognized and taken care of and their interests advanced". Disability-related issues were also captured under the 2017 MHS and the 2017/18 MICS survey by adopting the Washington Group on Disability Statistics' questionnaire [47]. Similarly, the RHPS [41] was primarily concerned with birth control for women who were "mentally disabled". The guidelines even specify that family-planning decisions for the mentally ill should be determined by their relatives. Surprisingly, the 2014 GDHS [50] which is the 6th version since its inception in the 1980s, seeks to monitor public health related issues in LMICs including Ghana, did not capture any issue related to disability. This is another evidence that the attention given to disability-related issues are cursory in most of the health policies/reports implemented in Ghana.

\subsection{Policy Objectives}

In general, almost all included policies had both general and specific objectives. Most of the objectives were qualitative without timelines and specific targets. However, the AHSPS and FPCIP had quantitative objectives that had timelines attached to achieve their desired objectives. The FPCIP [49] for example, had a quantitative objective of increasing the modern contraceptive use among currently married women from $22.2 \%$ to $29.7 \%$ by 2020 and to $33 \%$ for all contraceptive methods in 2020. With unmarried sexually active women, the policy seeks to increase the modern contraceptive prevalence from $31.7 \%$ to $40 \%$ by 2020 and up to $50 \%$ for all methods. Examples of the qualitative objectives can be found in the NHP [38], such as to strengthen the healthcare delivery system to be resilient; to encourage the adoption of healthy lifestyle; to improve the physical environment; to improve the socio-economic status of the population; and to ensure sustainable financing for health. The 2014 GDHS [50] (p. 5) also had this objective "to provide recent and reliable information on fertility, family planning, infant and child mortality, maternal and child health, and nutrition". The 2007 RHSP [41] objective was to "improve the health and quality of life of persons of reproductive age and newborn children by providing high-quality reproductive health services". Its strategic objectives focused on reducing maternal and neonatal morbidity and mortality, promoting and increasing access to quality reproductive health services, and increasing contraceptive prevalence and access. Most of the objectives did not capture PWDs (e.g., National HIV and AIDS, STI policy, DVR, NSA, GDHS, and GMHS). Some of the policy's aims were explicit and clear on how to improve and include PWDs in their programming [46]. For example, the gender policy and the NPP had specific objectives for PWDs. The NGP [46] (p. vii) indicates that:

"... The overarching goal of this Policy is to mainstream gender equality concerns into the national development processes by improving the social, legal, civic, political, economic and socio-cultural conditions of the people of Ghana particularly women, girls, children, the vulnerable and people with special needs; persons with disability and the marginalized".

\subsection{Scope of Activities}

This refers to the various activities to be carried out to ensure that the objectives of the policy are achieved. In general, all of the policies defined a wide range of operations to achieve their goals. Apart from the nationally representative surveys (GDHS, MHS, MICS and DVR), some of the policies had specific activities. For example, the RHSP [41] outlined the following scope of activities: information, education and communication, behavioural change, communication, improving access to maternal healthcare services (such as antenatal care, skilled delivery and post-natal care), promotion of access to contraceptive prevalence, and using community based volunteers. The AHSPS [40], also outlined the use of social- and behavioural communication-change strategies, ensure increase in access to healthcare for adolescents, and training (needs assessment of staff, and capacity building of staff). Considering this, some of the policies only captured a few specific activities to 
be carried out on PWDs. The NPP [39], for example, stated that it will employ the use of education, form a national coordinating committee on disability, enact laws and enforce existing ones, and provide assistance to PWDs. In addition, Section 5.9 of the NPP [39] (p. 27) also seeks to ensure that it creates opportunities for participation. It states that "To promote sound welfare programmes that would take care of the special needs of the youth, the aged, persons with disabilities and other vulnerable groups".

\subsection{Financial and Human Resources}

In this review, financial resources include monetary incentives, while the human resources are those that are required to aid the implementation and execution of the issues outlined. Human resources are needed to develop the policies and implementation of all the issues. Financial resources are needed to pay the human resources that help with the execution of policies. Some of the policies like the FPCIP [49], NHIP [45], and RHSP [41] specified the exact monetary values required to carry out their activities. However, they did not indicate how PWDs will also serve as human resources in the formulation or the execution of the specific activities of the policies.

\subsection{Monitoring and Evaluation Plan}

This review also investigated if the policies/reports had a monitoring and evaluation mechanism and performance indicators, and if so, whether these indicators were clear and related to the policies' objectives or not. The 2014 GDHS, 2017 MHS, MICS, DVR, COC and NSA did not have any monitoring and evaluation plans because the majority were reports released periodically. For example, the 2014 GDHS is the 6th version of the demographic and health survey report since its inception in 1988. The 2017 MHS is also the second version of the maternal health survey after the first one was conducted in 2007. The remaining policies had specified monitoring and evaluation plans. Some had performance indicators such as the RHSP [41], NHP [38], and FPCIP [49]. For example, the NHP [38] indicated that the "monitoring of the progress and achievement of the health outcomes will be routine and continuous (quarterly, half-yearly and annual)".

In the NPP [39] (p. 46), monitoring and evaluation was also captured as:

"The importance of reliable and timely collection of demographic data for formulation, monitoring and evaluation of development programmes cannot be over emphasized. Different strategies such as timely collection of data, strengthening the capacity of the Ghana health service and all training and research institutions and the enactment of laws governing the registration of vital events".

The NGP [46] also stated that "Monitoring and Evaluation shall be an essential strategy in the implementation of the NGP". In terms of disability-related objectives, most of the policies did not clearly specify how the monitoring of those objectives in PWDs will be conducted. For example, the NGP [46] (p. 45) stated thus: "Monitoring and Evaluation processes shall be put in place for each core component of the policy commitments to determine effectiveness, efficiency, economy, and equity compliance. These will be done via monthly update, 6 monthly monitoring and quality assurance monitoring and annual performance reviews". It did not indicate how the PWDs related objectives will be monitored and evaluated. This is not different from what was captured in the AHSPS [40]. All the performance indicators failed to specify that of the PWDs.

\subsection{Level of Coordination and Collaboration}

The level of cooperation and collaboration on various policies was also captured in this review. The bulk of the policies partnered with a variety of multi-national and non-governmental organisations such as United States Agency for International Development (USAID), UNFPA, European Union, WHO, UNICEF, UKAID and the National Commission for PWDs. For example, the DHS [50], MHS [51], and MICS [47] collaborated with organisations such as GSS and GHS. Financial support for the survey was provided by international organisations, such as the USAID, Global Fund, UNICEF and the Government 
of Ghana. Generally, apart from the COC [43] and the NSA [44], all the policies recognised the importance of coordination and collaboration. As this aided in the smooth delivery and achievement of policy objectives, most of these organisations also helped with the provision of human and financial resources. In the AHSPS [40], collaboration and coordination was well-captured as a strategic objective to "promote partnership and inter-sectoral collaboration among adolescent and youth groups, relevant institutions and communities in the provision and utilization of Adolescent and Youth Responsive Health Service" [40] (pp. 28-29).

\subsection{Level of Disability Involvement}

At each stage of the policy-making process, the extent and level of disability participation were reviewed (formulation, implementation plan, monitoring and evaluation). Only eight (AHSPS, NHP, MHS, MICS, NGP, NPP, COC, and NHISP) of the 15 policies involved PWDs. Assessment of policies that captured issues relating to disability such as the 2017 GMS, MICs, AHSPS and NGP, indicated that only the GP included an organisation on persons with disabilities in the drafting of the policy. In the two other documents (2017 MHS and the 2017/2018 MICS), disabled people were only part of the participants in their study either directly or indirectly [47].

With direct involvement, the PWDs were considered as a key population in the policy design. Typical examples include the AHSPS, NPP and NGP. The NPP [39] (p. 7) states that

"The issue of disability in our society also deserves serious consideration. Although official figures of registered persons with disabilities in the country in 1990 was 47, 397, it is believed, that unofficial figures might be well over 1 million. The proper sustenance and rehabilitation of these disabled persons demands serious attention".

The AHSPS [40] (p. 23) also included disability issues directly, and this is captured under the strategic objective as "improve access to specified packages of health services that are of high quality, gender sensitive, disability-responsive in an appropriate environment at all levels". However, various issues also demand attention to ensure that adolescents with disabilities are adequately considered. For example, the policy's goal is to provide a systematic method to understanding and prioritizing adolescent health needs, as well as planning, monitoring, and evaluating adolescent health programs. Yet, it fails to address the health requirements of adolescents with impairments. It also demonstrates that teenagers and young girls without disabilities are more aware of contraception and utilise it, but there is no mention of the situation among adolescents and young girls with disabilities. In addition, the policy stated that the majority of Ghanaians lack access to reproductive health counselling, contraception, and safe abortion services, and that even in areas where these services are available, young people's access is limited due to distance to services, provider bias, inconvenient opening hours, legal restrictions and negative labelling. The guidelines didn't specify how negative attitudes and distance from services influence adolescents and young people with disabilities' health-seeking behaviours.

In general, the health needs of PWDs are inadequately captured in Ghana's health policies, based on the 15 policies examined. Rather, disability is referenced only in passing in selected sections. Even in policies that claim to add disability-related issues, disability only appeared in a few sections. For example, the NHP mentioned disability in only six pages $(10,11,14,18,20$, and 30$)$ out of the 46 pages of the document. This gives the impression that disability-related issues were not considered as integral components of the policy. Furthermore, prior to the AHSPS [40], PWDs were labelled as minorities, vulnerable persons, or those with compounded vulnerabilities. This meant that a thorough assessment of the requirements of PWDs as well as appropriate interventions were not carried out.

In the NPP [39] for example, while women and teenagers were treated individually, PWDs and the aged were lumped together and given the same treatment, despite the fact that their problems and needs are not always the same. The Ghana National HIV / AIDS and STI Policy [27] for example, solely captured PWDs in terms of social protection. Meanwhile they are already a marginalized group that experiences various obstacles and challenges, including sexual violence, which can easily predispose them to STIs including HIV and 
AIDs. Therefore, the SRH- and STIs-prevention needs of PWDs were not considered. The 2014 GDHS [50] for example, looked at the links between reproductive health and a range of variables. It provides a wide picture of the demographic characteristics and health condition of Ghana's general population. Nonetheless, it failed to add PWDs. In terms of STIs and unwanted pregnancies, the NSA supplied extensive data on adolescent risk-taking and health-seeking behaviour. The document examined a variety of issues, including social, behavioural, and economic factors that could increase the vulnerability of adolescents to SRH problems, knowledge about prevention methods, trusted sources of information, and healthcare and barriers to putting what they learned into practice. Nevertheless, adolescents with disabilities were missing out of these measures. In NRHPS [48], the emphasis is mainly on birth control for "mentally disabled" women. The regulation specifies that the next-of-kin should make decisions on family planning for the "mentally disabled". In as much as this is a way to ensure that persons with mental disabilities use family planning methods, this is unfortunate because in addition to mental disability, there are various forms of disabilities including visual, hearing, and physical disabilities.

There are also instances in which PWDs are considered in the policies/ reports, but with a negative connotation. For example, the COC [43] used the world "female idiot" as a justifiable reason to terminate a pregnancy. The specific reason why the pregnancy can be terminated is not well articulated. Another major issue about the inclusion of PWDs in policies is the accessibility of the policies to the general population and PWDs in particular. Although all the policies that were captured in this review were online, none of them explained how efforts are being made to ensure that PWDs get easy access to these policies in different formats, such as braille or audio versions in the languages they understand. This is peculiar to those who can read, but what about those who cannot read and write?

\section{Discussion}

This review assessed the inclusiveness of PWDs in Ghanaian health policies and reports. Almost all the fifteen policies were backed by political recognition, but the level of inclusion of disability issues was low. The use of both financial and human resources to achieve stated objectives was observed in all the policies. However, only a few of these policies described the costs associated with various activities to be carried out. Some of the policies also acknowledged the important roles of collaboration and coordination. Previous studies $[25,54,55]$ indicated that political recognition is a key factor in policy development and implementation. Similarly, Birdthistle and Vince-Whitman [56] noted that clear national policies and documents were often critical to the success of SRH programs.

Despite the political recognition in these policies, the level of involvement of PWDs remains low. Only eight of the policies considered disability-related issues with varied levels of inclusiveness. There were variations in the attention given to them. Some of the policies treated disability and the aged as a similar group, while others considered disability in a narrow sense of "mental disability" and some connoted disability in a negative sense [43], which is against the article 12.2 of the UNCRPWDs [4] (pp. 10-11). This confirms a previous study by Mprah et al. [29] who focused on only SRH policies and found that the inclusion of PWDs was very low. Mprah et al. [29] opined that several reasons account for the neglect of PWDs in some of the policies in Ghana. Despite the bad treatment meted out to PWDs being recognised as criminal offences under the Ghanaian Constitution and the Persons with Disability Act 715, negative perceptions about PWDs still persist. The policies were designed without the involvement of PWDs and this is a clear reflection of such negative attitudes and perceptions. They also added that PWDs were not regarded as either contributors to the population's problem or economically productive, and some of the policies and programs were donor funded with limited government funding. Nonetheless, in this current review, most of the policies had political recognition, which presupposes that the government has control over the enactment and implementation of the various policies. Relatedly, Abdul Karimu [30] also reviewed the 2016 AHSPS and concluded that there are various loopholes that need to be considered 
in the policy. For example, she stated that contraception access and counselling are not well captured among adolescents with disabilities. The findings are also similar in other parts of the geographic region. For instance, Lang et al. [28] also indicated that the level of inclusion of disability issues in the African Union policies is very low and this is also evident in all social protection programs in LMICs [57].

Juxtaposing the findings of this current study with some health policies in other parts of the world exhibit some discrepancies and similarities. For example, Rwanda's health sector policy [58] has indicated that access to health services for PWDs has improved. This is corroborated by a review conducted by Njelesani et al. [59] affirmed there has been realisation of the rights of PWDs in Rwanda, pertinent challenges still exist within their policies. Their health sector policy has outlined that specific protocols for disability-friendly services shall be established in all hospitals, and that consultation services will be available at health-centre level for this vulnerable group. Although this is not specific enough, it has stipulated what will be done at the health-facility level, which was not considered in the 2020 NHP of Ghana [38]. In South Africa, the inclusiveness of disability issues in their national health promotion policy and strategy (2016-2019) [60] is not too different from that which pertains to the NHP [38]. In the South African Health policy, in terms of the target audience, PWDs have been captured under the term marginalised population. As this is a great step, the needs of PWDs are peculiar and for that matter, they should not be lumped with a general group. In terms of the policy formulation, although various disability organisations such as South African Disability Alliance, Disabled People South Africa, and National Council for and of people with disabilities exist within the country, none was mentioned as a partner who assisted in the development of the policy. Similarly, the National Health Promotion Policy of Nigeria, 2019 [61] (p.7) has also captured disability-related issues. For example, the action point 1, under the objective of fostering health promotion interventions to address the social determinants of health including the reduction of inequities, and tackling priority burden of diseases in Nigeria has featured PWDs. The document also failed to mention the involvement of specific disability organisations or groups that aided in the development of the revised health policy. In Namibia, the national health policy framework, 2010-2020 [62] (p.13-14), also captured PWDs, nonetheless mental health and disability issues were lumped together. This is also an indication that disability-related concerns have not been given ample attention in some of the countries also within the sub-region. This reiterates the findings of the WHO [3] that PWDs have been neglected in most policies and interventions globally.

Despite this, the development of policies by international organisations such as the WHO make conscious efforts to include PWDs. A typical example is the WHO inclusive health service toolkit for the WHO western pacific region. In the formulation of the documents various PWDs and their organisations were actively involved in the development and design of the toolkit. For instance it states PWDs and their organization, the Pacific Disability Forum and Inclusive Development and Empowerment Agenda all participated in the development of the policy [7]. This is also similar to the World Report on Disability [1] that was commissioned in 2011. These are examples worthy of emulating in terms of policy formulation relating to PWDs.

Strengths and Limitations

The use of a validated and well-known model to analyse the policies/reports on the inclusion of disability concerns in Ghana's health policies is one of the review's key strengths. The review also considered multiple sources, such as official government websites, those of various ministries, and Google scholar to identify as many policies/reports as possible. However, the review was restricted to only Ghana and to policies/reports that were published online. We also recognize that the policies may not fully represent all procedures and actions involved in the policy formulation process. Furthermore, the scoping review is constrained in its reliance on secondary data derived solely from documents. Primary data gathering from individuals who designed the policies and initiatives, policy implementers and various beneficiaries such as PWDs, would have improved the findings. 


\section{Conclusions}

This review was conducted to assess the level of inclusiveness of disability issues in health policies/reports in Ghana. Although most of the policies reviewed had political recognition, the level of inclusion of PWDs in policy development and objectives is very low. Specifically, while some of the documents analysed made mention of disability and the significance of addressing disability concerns, many others did not. The definition of disability was also limited in some of the policies. The monitoring and evaluation aspect of some of the policies also failed to consider PWDs. To achieve the United Nations' SDG 10 of 'leaving no one behind' as well as the provisions in the UNCRPWDs and the Disability Act 715 of Ghana, it is recommended that subsequent health policies/reports engage PWDs right from the design stage to the implementation of the policies. This will ensure that PWDs are able to air their grievances and able to make cogent policy inputs borne out of their personal experiences. Their views on how best to implement the policies and the traps to avoid in overcoming barriers to successful outcomes will also help with compliance and contribute adequately to health policies in Ghana. In addition, there should be mechanisms in place to sensitise PWDs on the policies that are available and how they can access those policies. It is imperative to clearly specify budgetary and human resource provision and the plans for monitoring and evaluation of disability-related targets. The research methodology employed in the current study could also be applied to analyse other sectorial policies, in Ghana and other countries, on the inclusiveness of disability issues.

Supplementary Materials: The following are available online at https:/ / www.mdpi.com/article/ 10.3390/disabilities1030019/s1, S1: Preferred Reporting Items for Systematic reviews and MetaAnalyses extension for Scoping Reviews (PRISMA-ScR) Checklist.

Author Contributions: Writing—original draft preparation, A.-A.S.; writing—review and editing, A.-A.S., B.S.M.-A., K.M.-R., A.E.O.M.-A. and T.I.E. All authors approved it for publication. All authors have read and agreed to the published version of the manuscript.

Funding: This research received no external funding.

Institutional Review Board Statement: Not applicable.

Informed Consent Statement: Not applicable.

Data Availability Statement: Not applicable.

Conflicts of Interest: The authors declare no conflict of interest.

\section{References}

1. WHO. World Report on Disability; WHO: Geneva, Switzerland, 2011; Available online: https://apps.who.int/iris/handle/10665/ 44575 (accessed on 1 June 2021).

2. Mitra, S.; Yap, J. The Disability Data Report: Disability Data Initiative; Fordham Research Consortium on Disability: New York, NY, USA, 2021; pp. 1-69.

3. WHO. Disability and Health. Available online: https://www.who.int/news-room/fact-sheets/detail/disability-and-health (accessed on 1 June 2021).

4. UN. Convention on the Rights of Persons with Disabilities; United Nations: New York, NY, USA, 2006.

5. Ghana Disability Data Disaggregation Pilot Project: Results of Integrating Disability into Routine Data Collection Systems; Sightsavers: Chippenham, UK, 2018.

6. OHCHR. Status of Ratification Interactive Dashboard; Office of the High Commissioner for Human Rights Retrieved: Geneva, Switzerland, 2021; Available online: https:/ /indicators.ohchr.org/ (accessed on 1 May 2021).

7. WHO. Disability-Inclusive Health Services Toolkit: A Resource for Health Facilities in the Western Pacific Region; World Health Organization Regional Office for the Western Pacific: Manila, Philippines, 2020; pp. 1-234.

8. UN. Transforming Our World: The 2030 Agenda for Sustainable Development; United Nations: New York, NY, USA, 2015.

9. Asuman, D.; Ackah, C.G.; Agyire-Tettey, F. Disability and household welfare in Ghana: Costs and correlates. J. Fam. Econ. Issues 2020, 2020, 1-7.

10. Gichane, M.W.; Heap, M.; Fontes, M.; London, L. "They must understand we are people": Pregnancy and maternity service use among signing Deaf women in Cape Town. Disabil. Health J. 2017, 10, 434-439. [CrossRef] [PubMed]

11. Madans, J.H.; Loeb, M.E.; Altman, B.M. Measuring disability and monitoring the UN Convention on the Rights of Persons with Disabilities: The work of the Washington Group on Disability Statistics. BMC Public Health 2011, 11, 1-4. [CrossRef] 
12. SSharma, S.; Sivakami, M. Sexual and reproductive health concerns of persons with disability in India: An issue of deep-rooted silence. J. Biosoc. Sci. 2018, 51, 225-243. [CrossRef] [PubMed]

13. Esmail, S.; Darry, K.; Walter, A.; Knupp, H. Attitudes and perceptions towards disability and sexuality. Disabil. Rehabil. 2010, 32, 1148-1155. [CrossRef] [PubMed]

14. Badu, E.; Gyamfi, N.; Opoku, M.P.; Mprah, W.K.; Edusei, A.K. Enablers and barriers in accessing sexual and reproductive health services among visually impaired women in the Ashanti and Brong Ahafo regions of Ghana. Reprod. Health Matters 2018, 26, 51-60. [CrossRef] [PubMed]

15. Groce, N.; Kett, M.; The Disability and Development Gap. Leonard Cheshire Disability and Inclusive Development Centre Working Paper Series. 2013. Available online: https://www.academia.edu/download/51014735/WP21_Disability_and_ Development_Gap.pdf (accessed on 1 May 2021).

16. Badu, E.; Opoku, M.P.; Appiah, S.C.Y. Attitudes of health service providers: The perspective of people with disabilities in the kumasi metropolis of ghana. Afr. J. Disabil. 2016, 5, 1-8. [CrossRef] [PubMed]

17. Badu, E.; Mensah, I.; Gyamfi, N.; Agyei-Okyere, E.; Abodey, E.; Adusei-Nkrumah, J. Knowledge and sources of accessing sexual and reproductive health information among visually impaired women in Ghana. BMC Res. Notes 2019, 12, 1-8. [CrossRef]

18. Saulo, B.; Walakira, E.; Darj, E. Access to healthcare for disabled persons. How are blind people reached by HIV services? Sex. Reprod. Healthc. 2012, 3, 49-53. [CrossRef]

19. Burke, E.; Kébé, F.; Flink, I.; Van Reeuwijk, M.; Le May, A. A qualitative study to explore the barriers and enablers for young people with disabilities to access sexual and reproductive health services in Senegal. Reprod. Health Matters 2017, 25, 43-54. [CrossRef]

20. Holdsworth, E.; Trifonova, V.; Tanton, C.; Kuper, H.; Datta, J.; MacDowall, W.; Mercer, C. Sexual behaviours and sexual health outcomes among young adults with limiting disabilities: Findings from third British National Survey of Sexual Attitudes and Lifestyles (Natsal-3). BMJ Open 2018, 8, e019219. [CrossRef]

21. Frohmader, C.; Ortoleva, S. The Sexual and Reproductive Rights of Women and Girls with Disabilities. 2013. Available online: https: / / scholar.google.com/scholar?hl=en\&as_sdt=0\%2C5\&q=21.\%09Frohmader \%2C+C. $\% 3 \mathrm{~B}+\mathrm{Ortoleva} \% 2 \mathrm{C}+\mathrm{S}$. + The+ Sexual+and+Reproductive+Rights+of+Women+and+Girls+with+Disabilities \%3B+Publisher\%3A+city \%2C+country\%2C+2013 .\&btnG $=$ (accessed on 1 May 2021).

22. Bremer, K.; Cockburn, L.; Ruth, A. Reproductive health experiences among women with physical disabilities in the Northwest Region of Cameroon. Int. J. Gynecol. Obstet. 2009, 108, 211-213. [CrossRef]

23. UNFPA. A Deeper Silence: The Unheard Experiences of Women With Disabilities and their Sexual and Reproductive Health Experiences: Kiribati, the Solomon Islands and Tonga; United Nations Population Fund Pacific Sub-Regional Office: Suva, Fiji, 2013.

24. Addlakha, R.; Price, J.; Heidari, S. Disability and sexuality: Claiming sexual and reproductive rights. Reprod. Health Matters 2017, 25, 4-9. [CrossRef] [PubMed]

25. Ahinkorah, B.O.; Kang, M.; Perry, L.; Brooks, F. Prevention of adolescent pregnancy in Anglophone sub-Saharan Africa: A scoping review of national policies. Int. J. Health Policy Manag. 2020. [CrossRef] [PubMed]

26. Agbenu, I. Evaluation of the Implementation of Adolescent Health Service Policy and Strategy (2016-2020) in the Tema Metropolis; University of Ghana: Accra, Ghana, 2019; pp. 1-157.

27. GAC. National HIV and AIDS, STI Policy; Ghana AIDS Commission: Accra, Ghana, 2013; pp. 1-67. Available online: https: / / www.google.com/url?sa=t\&rct=j\&q=\&esrc=s\&source=web\&cd=\&cad=rja\&uact=8\&ved=2ahUKEwjBosL4 8IfxAhWIsRQKHWCzCKwQFjAAegQIAhAD\&url=https\%3A\%2F\%2Fwww.healthpolicyproject.com\%2Fpubs\%2F153 _Policyfinal.pdf\&usg=AOvVaw3h4sEOigw2Vj4R6OIoe5Tj (accessed on 1 May 2021).

28. Lang, R.; Schneider, M.; Kett, M.; Cole, E.; Groce, N. Policy development: An analysis of disability inclusion in a selection of African Union policies. Dev. Policy Rev. 2018, 37, 155-175. [CrossRef]

29. Kwadwo, W.; Anafi, P.; Sekyere, F.O. Does disability matter? Disability in sexual and reproductive health policies and research in Ghana. Int. Q. Community Health Educ. 2014, 35, 21-35. [CrossRef] [PubMed]

30. Karimu, A.T.F.A. Disabled persons in Ghanaian health strategies: Reflections on the 2016 adolescent reproductive health policy. Reprod. Health Matters 2018, 26, 20-24. [CrossRef]

31. Mfoafo-M'Carthy, M.; Grischow, J.D.; Stocco, N. Cloak of invisibility: A literature review of physical disability in Ghana. SAGE Open 2020, 10. [CrossRef]

32. Hashemi, G.; Kuper, H.; Wickenden, M. SDGs, Inclusive health and the path to universal health coverage. Disabil. Glob. South 2017, 4, 1088-1111.

33. Jamali, M.Z. Disability Measurement and Uptake of Sexual and Reproductive Health Services in Malawi. Ph.D. Thesis, University of Southampton, Southampton, UK, 2020.

34. Arksey, H.; O'Malley, L. Scoping studies: Towards a methodological framework. Int. J. Soc. Res. Methodol. 2005, 8, 19-32. [CrossRef]

35. Jones, C.M.; Gautier, L.; Ridde, V. A scoping review of theories and conceptual frameworks used to analyse health financing policy processes in sub-Saharan Africa. Health Policy Plan. 2021. [CrossRef] [PubMed] 
36. Calves, A. Assessing Adolescent Reproductive Health Policies and Programs. In Policy Working Paper Series; USAID: Washington, DC, USA, 2002; pp. 1-33. Available online: https:/ /scholar.google.com/scholar?hl=en\&as_sdt=0\%2C5\&q=Calves $\% 2 C+A .+\% 28$ $2002 \% 29 .+$ Assessing+adolescent+reproductive + health+policies+and+programs.+Retrieved+from + Washington $\% 2 \mathrm{C}+\mathrm{DC} \% 3 \mathrm{~A}+$ $\& b \operatorname{tnG}=($ accessed on 1 May 2021).

37. Tricco, A.C.; Lillie, E.; Zarin, W.; O’Brien, K.K.; Colquhoun, H.; Levac, D.; Moher, D.; Peters, M.D.J.; Horsley, T.; Weeks, L. PRISMA extension for scoping reviews (PRISMA-ScR): Checklist and explanation. Ann. Int. Med. 2018, 169, 467-473. [CrossRef] [PubMed]

38. MOH. National Health Policy: Ensuring Healthy Lives for All; Ministry of Health: Accra, Ghana, 2020; pp. 1-46. Available online: https: / / www.google.com/url?sa=t\&rct=j\&q=\&esrc=s\&source=web\&cd=\&cad=rja\&uact=8\&ved=2ahUKEwjXoLbz8 YfxAhVJ5uAKHQXZC0MQFjAAegQIAxAD\&url=https\%3A\%2F\%2Fwww.moh.gov.gh\%2Fwpcontent\%2Fuploads\%2F2020\% 2F07\%2FNHP_12.07.2020.pdf-13072020-FINAL.pdf\&usg=AOvVaw1G--5Y2AsC0xcGkqx6Dv_U (accessed on 1 May 2021).

39. GOG. National Population Policy (Revised Edition, 1994). 1994. Available online: https://www.google.com/url?sa=t\&rct=j\&q= \&esrc=s\&source=web\&cd=\&cad=rja\&uact=8\&ved=2ahUKEwjSb76qtPxAhWBoVwKHebHCCoQFjABegQIBBAD\&url=https\% 3A\%2F\%2Fnpc.gov.gh\%2Fwp-content\%2Fuploads\%2F2019\%2F01\%2FNational-Population-PolicyRevised-Edition-1994.pdf\& usg=AOvVaw0PI2IHJYnyOhqKL4RVxfGI (accessed on 1 May 2021).

40. GHS. Adolescent Health Service Policy and Strategy (2016-2020); Ghana Health Service: Accra, Ghana, 2020; Available online: https:/ /www. google.com/url?sa=t\&rct=j\&q=\&esrc=s\&source=web\&cd=\&ved=2ahUKEwiG3_zZydDyAhUmQEEAHYnzCRYQFnoECAQQAQ\& url=https \%3A\%2F\%2Fwww.afro.who.int \%2Fsites\%2Fdefault\%2Ffiles\%2F2017-10\%2FADOLESCENT\%2520HEALTH\%2520SERVICE\% 2520POLICY\%2520\%2520AND\%2520STRATEGY.pdf\&usg=AOvVaw3bBY-GRD6_kQnXb-4Bno9A (accessed on 1 May 2021).

41. GHS. Reproductive Health Strategic Plan 2007-2011. 2007. Available online: https://www.google.com/url?sa=t\&rct=j\& $\mathrm{q}=\&$ esrc $=s \&$ source=web\&cd=\&cad=rja\&uact=8\&ved=2ahUKEwiwsNyT9YfxAhUM0uAKHSZ3CykQFjABegQIBBAD\&url= http \%3A\%2F\%2Fwww.moh.gov.gh\%2Fwp-content\%2Fuploads\%2F2016\%2F02\%2FGHS-Reproductive-Health-Strategic-PlanFINAL.pdf\&usg=AOvVaw35xjp5uCQ-rpwmCmF-EKQ3 (accessed on 1 May 2021).

42. IDS; GSS and Associates. Domestic Violence in Ghana: Incidence, Attitudes, Determinants and Consequences. 2016. Available online: https: / / www.google.com/url?sa=t\&rct=j\&q=\&esrc=s\&source=web\&cd=\&cad=rja\&uact=8\&ved=2ahUKEwipnfKC9 ofxAhVuA2MBHdSvA6cQFjACegQIAxAE\&url=https\%3A\%2F\%2Fwww2.statsghana.gov.gh\%2Fdocfiles \%2Fpublications\% 2FDV_Ghana_Report_FINAL.pdf\&usg=AOvVaw2ybOzhhduAH5Td0CFYGk6T (accessed on 1 May 2021).

43. GOG. Criminal Code 1960 (ACT, 29). 1960. Available online: https: $/ /$ www.google.com $/$ url? sa=t\&rct=j\&q=\&esrc=s\&source= web\&cd=\&cad=rja\&uact=8\&ved=2ahUKEwiW4tKZq9PxAhXahVwKHQrrAewQFjADegQIBRAD\&url=https\%3A\%2F\% 2Fhome.gis.gov.gh\%2Fwp-content \%2Fuploads\%2F2020\%2F05\%2FCriminal-Offences-Act-1960-Act-29.pdf\&usg=AOvVaw1A7 R-yWgXvCFI5o2PodrGW (accessed on 1 May 2021).

44. Awusabo-Asare, K.; Abane, A.M.; Kumi-Kyereme, A. Adolescent Sexual and Reproductive Health in Ghana: A Synthesis of Research Evidence, Occasional Report; The Alan Guttmacher Institute: New York, NY, USA, 2004; Volume 13, pp. 1-37.

45. MOH. National Health Insurance Policy; MOH: Accra, Ghana, 2004; Available online: https:/ /www.google.com/url?sa=t\&rct=j\& $\mathrm{q}=\& \mathrm{esrc}=\mathrm{s} \&$ source=web\&cd=\&cad=rja\&uact=8\&ved=2ahUKEwiC_56nqNPxAhU1olwKHcHqA3QQFjAAegQIBRAD\&url= https \%3A\%2F\%2Fextranet.who.int\%2Fnutrition\%2Fgina\%2Fen\%2Fnode\%2F8121\&usg=AOvVaw2rz6rYKnEFrXhiKrJgkaOX (accessed on 1 May 2021).

46. GOG. National Gender Policy. 2015. Available online: https:/ /www.google.com $/$ url?sa=t\&rct=j\&q=\&esrc=s\&source=web\&cd= \&cad=rja\&uact=8\&ved=2ahUKEwiP0OnO94fxAhVR7OAKHXdAFoQFjAMegQIFRAD\&url=https\%3A\%2F\%2Fwww.mogcsp. gov.gh\%2Fmdocs-posts\%2Fnational-gender-policy\%2F\&usg=AOvVaw1qlYJiNL4OULY97kmD94tr (accessed on 1 May 2021).

47. GSS. Multiple Indicator Cluster Survey (MICS2017/18), Survey Findings Report; GSS: Accra, Ghana, 2018; Available online: $\quad$ https: / / www.google.com/url?sa=t\&rct=j\&q=\&esrc=s\&source=web\&cd=\&cad=rja\&uact=8\&ved=2ahUKEwiFp6 aTfxAhXgBmMBHfQABYkQFjABegQIBBAD\&url=https\%3A\%2F\%2Fwww.unicef.org\%2Fghana\%2Freports\%2Fghanamultiple-indicator-cluster-survey\&usg=AOvVaw2Qyn1V1D8SM9oRnT2AE6Ky (accessed on 1 May 2021).

48. MOH. National Reproductive Health Policy and Standards. 2003. Available online: https://www.google.com/url?sa=t\&rct=j\& $\mathrm{q}=\&$ esrc=s\&source=web\&cd=\&cad=rja\&uact=8\&ved=2ahUKEwij7_7BqNPxAhVVglwKHXysCNUQFjAKegQIMRAD\&url= https\%3A\%2F\%2Fwww.ghanahealthservice.org\%2Fdownloads\%2FNHI_policy\%2520framework.pdf\&usg=AOvVaw1MJE_i5 eIFLOz9YmRIBNOP (accessed on 1 May 2021).

49. GOG. Ghana Family Planning Costed Implementation Plan 2016-2020; Ghana Health Service: Accra, Ghana, 2015.

50. GSS; GHS; ICF. Ghana Demographic and Health Survey 2014; GSS, GHS, and ICF: Rockville, MD, USA, 2015.

51. GSS; GHS; ICF. Ghana Maternal Health Survey 2017; GSS, GHS, and ICF: Accra, Ghana, 2018.

52. Vaismoradi, M.; Turunen, H.; Bondas, T. Content analysis and thematic analysis: Implications for conducting a qualitative descriptive study. Nurs. Health Sci. 2013, 15, 398-405. [CrossRef] [PubMed]

53. Bloor, M.; Wood, F. Phenomenological methods. Keywords Qual. Methods 2006, 56, 128-130.

54. Dye, T.R. Understanding Public Policy; Pearson: Upper Saddle River, NJ, USA, 1998.

55. Stover, J.; Johnston, A.; The Art of Policy Formulation: Experiences from Africa in Developing National HIV. AIDS Policies. 1999. Available online: http:/ / www.plicyprogect.com/pubs/occasional/op-03PDF (accessed on 1 May 2021).

56. Birdthistle, I.; Vince-Whitman, C. Reproductive Health Programs for Young Adults: School-Based Programs; Pathfinder International, Focus on Young Adults Project: Watertown, MA, USA, 1997. 
57. Walsham, M.; Kuper, H.; Banks, L.M.; Blanchet, K. Social protection for people with disabilities in Africa and Asia: A review of programmes for low-and middle-income countries. Oxf. Dev. Stud. 2018, 47, 97-112. [CrossRef]

58. MOH. Health Sector Policy; Ministry of Health: Kigali, Rwanda, 2015; pp. 1-41. Available online: https://www.moh.gov.rw/ news-detail/health-sector-policy-2015 (accessed on 1 July 2021).

59. Njelesani, J.; Siegel, J.; Ullrich, E. Realization of the rights of persons with disabilities in Rwanda. PLoS ONE 2018, 13, 1-15. [CrossRef]

60. DOH. National Health Promotion Policy and Strategy, 2015-2019; Department of Health: Pretoria, South Africa, 2015; Available online: https:/ / www.health-e.org.za/wp-content/uploads/2015/09/The-National-Health-Promotion-Policy-and-Strategy.pdf (accessed on 1 July 2021).

61. FMOH. National Health Promotion Policy; FMOH: Abuja, Nigeria, 2019; pp. 1-33. Available online: https://www. google.com / url? sa=t\&rct=j\&q=\&esrc=s\&source=web\&cd=\&cad=rja\&uact=8\&ved=2ahUKEwiR4abcltLxAhXBQkEAHd1 nBIgQFjABegQIBRAD\&url=https\%3A\%2F\%2Fwww.health.gov.ng\%2Fdoc\%2FNHPP_2019.pdf\&usg=AOvVaw3nsSO9pb2 yHTuomf_b_0ez (accessed on 1 July 2021).

62. MHSS. National Health Policy Framework, 2010-2020. In Ministry of Health and Social Services, Windhoek, Namibia; 2010; pp. 1-40. Available online: https / /www.google.com/url?sa=t\&rct=j\&q=\&esrc=s\&source=web\&cd=\&ved=2ahUKEwjI1 YHD48vyAhVymVwKHZGbBPEQFnoECAIQAQ\&url=https\%3A\%2F\%2Fextranet.who.int\%2Fcountryplanningcycles\% 2Fsites\%2Fdefault\%2Ffiles\%2Fcountry_docs\%2FNamibia\%2Fnamibia_national_health_policy_framework_2010-2020.pdf\& usg=AOvVaw3pmJVGOWC_V4f-iqW67SA0 (accessed on 1 July 2021). 